\title{
Designing Shared Gaze Awareness for Remote Collaboration
}

\author{
Jerry Li \\ Northwestern University \\ Evanston, IL 60208, USA \\ jiamingli2017@u.northwestern.edu
}

\section{Mia Manavalan}

Northwestern University

Evanston, IL 60208, USA

MiaManavalan2016@u.northwestern.edu

\section{Sarah D'Angelo}

Northwestern University

Evanston, IL 60208, USA

sdangelo@u.northwestern.edu

\section{Darren Gergle}

Northwestern University

Evanston, IL 60208, USA

degergle@northwestern.edu

\begin{abstract}
In this project, we evaluate two different methods for highlighting shared gaze across two tasks with different collaborative properties. There are many factors to consider when designing shared gaze representations such as how much information to display and when to provide it so that it will be most useful. Unlike other non-verbal forms of communication such as deictic gesturing, gaze is not always intentionally communicative and therefore we need to think critically about when and how to display it. For each task, participants saw their partner's gaze displayed continuously, emphasized either by previous fixation points or extended fixations. We discuss our findings and present design implications for shared gaze awareness based on interaction traces and interviews with participants.
\end{abstract}

\section{Author Keywords}

Eye-tracking; design; computer supported collaborative work.

Permission to make digital or hard copies of part or all of this work for personal or classroom use is granted without fee provided that copies are not made or distributed for profit or commercial advantage and that copies bear this notice and the full citation on the first page. Copyrights for third-party components of this work must be honored. For all other uses, contact the Owner/Author.

Copyright is held by the owner/author(s)

CSCW '16 Companion, February 27 - March 02, 2016, San Francisco, CA USA

ACM 978-1-4503-3950-6/16/02

http://dx.doi.org/10.1145/2818052.2869097

\section{ACM Classification Keywords}

H5.3. Information interfaces and presentation (e.g.

$\mathrm{HCI}$ ): Group and organizational interfaces - collaborative computing, computer-supported collaborative work 


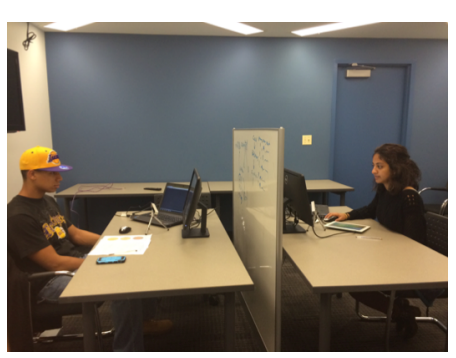

Figure 1: Experiment setup

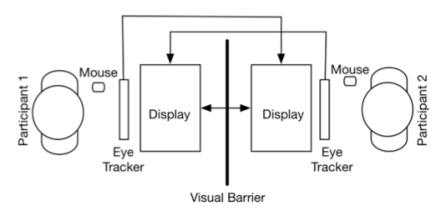

Figure 2: Apparatus

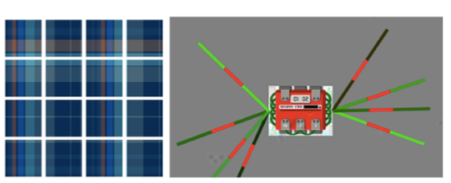

Figure 3: Example stimuli of puzzle and bomb diffusion tasks

\section{Introduction}

Eye tracking technology is increasingly accessible with modern advancements in webcam based systems ${ }^{1}$ and affordable remote eye trackers ${ }^{2}$. These advances encourage the use of gaze awareness features in a variety of remote collaborative settings such as online education and medical training $[4,5]$. However, gaze information can be a noisy communicative signal, therefore it is important to understand how to design gaze information to best support collaboration.

In this project, we evaluate the design of shared gaze information for remote collaborative work. We designed two methods for highlighting a partner's gaze information and examine its use across two different collaborative tasks. The tasks are designed to highlight the potential value of shared gaze. The stimuli are linguistically complex with difficult to describe colors and dynamic stimuli (see figure 3 ). After participants complete the tasks, they are interviewed about their perceptions of the different representations for highlighting gaze. Our results suggest that the task structure influences the value of seeing a partner's gaze, and in response we reflect on how gaze should be best designed to facilitate collaboration.

\section{Related Work}

Previous work on collaborative conceptual learning and collaborative search tasks have shown that sharing gaze information improves pair performance $[1,3]$. Additionally, in asynchronous collaborative tasks such as code review, studies have shown that sharing gaze paths can influence how people parse the code [6].

\footnotetext{
${ }^{1}$ http://www.pygaze.org/

${ }^{2}$ https://theeyetribe.com/
}

However, the question of how shared gaze awareness should be designed given the particular task structure has not been directly addressed.

\section{Method}

Participants

8 University students participated in the study and were paired with a confederate to complete the collaborative tasks. Individuals with colorblindness were excluded. Consent was obtained and participants received $\$ 10$ or course credit.

\section{Procedure}

Pairs interact in a pseudo-remote configuration with a visual barrier allowing them to communicate verbally (figures $1 \& 2$ ). Participant's eye gaze is calibrated at the beginning of the study using a 9-point calibration and is recalibrated as needed in order to maintain accuracy. Participants are informed they will be completing two tasks: a puzzle task and a bomb diffusion task.

Participants complete each task twice with the different representations for highlighting the partner's gaze.

Participants are told they will be shown their partner's gaze. Time and score are displayed on screen for both tasks to encourage fast and accurate performance. After completing the task, a researcher asks the participants a series of questions about the representations used for highlighting gaze. The participant's responses are transcribed for analysis.

\section{Apparatus}

In this experiment we use two Eyetribe remote eye trackers and two 20" Dell monitors with wireless mice (figure 2). The eye trackers record participant's gaze and our software displays the visual representation of gaze coordinates on the partner's screen in real time. 


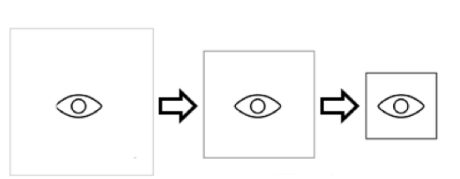

Figure 4: Zoom focus

"I tried to use the focus box a few times. It was difficult because I couldn't tell if it was activating and I didn't want to waste time".

- Subject \#4

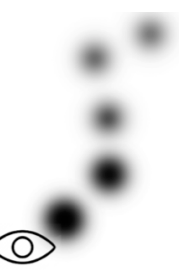

\section{Figure 5: Gaze trail}

"I could kind of see if you looked at one place to another, I could tell that you were trying to connect those two."

- Subject \#6

"I got more comfortable [using gaze] as I learned" - Subject \#3

\section{Gaze Representations}

The exact gaze coordinates are represented on screen by an icon resembling an eye ( $50 \times 50$ pixels). This is continuously displayed on the screen and highlighted in two different ways:

Zoom Focus: When a fixation occurs in the same location for $0.5 \mathrm{~s}$, a square $(1000 \times 1000$ pixels $)$ appears and zooms in to the precise location of the fixation represented by the gaze cursor (figure 4).

Gaze Trail: Previous fixations points are displayed with the most recent gaze locations depicted in black and the older gaze locations fading out after 2 s (figure 5 ).

Tasks

In the collaborative puzzle task participants work together to complete a $4 \times 4$ plaid pattern with 16 individual pieces (figure 6). All of the pieces are unique but they are visually complex and difficult to differentiate [2]. Additionally, the pieces can only be moved when both participants are simultaneously selecting two pieces to combine. If they attempt to make an incorrect combination they lose points. These aspects of the task were designed to increase the utility of shared gaze.

In the leader/follower bomb diffusion task (figure 7), a bomb is represented on screen with eight moving wires (four on the left and four on the right). The wires are varying shades of green and continually moving. These task attributes prevent the easy use of location and color descriptions to refer to specific wires. The director has the solution for the correct order to cut the wires and instructs their partner to click a specific wire to cut. If the incorrect wire is "cut" the bomb explodes and the task restarts.

\section{Results}

Shared gaze awareness was a novel feature and initially confusing for all of the participants. As the study progressed participants adapted to use the gaze information to simplify the task by producing more deictic references (e.g., "this piece over here").

Zoom Focus: We designed the zoom focus representation to highlight the gaze display with selective automatic detection based on fixation duration. Long fixations could indicate areas of interest that would be relevant to the collaborative partner. However, most participants found it to be too cognitively demanding and expressed that the lack of feedback made it unclear if they were successfully signaling. Additionally, the 500-millisecond delay for the zoom to occur was enough delay to make some references ambiguous.

Gaze Trail: The gaze trail was designed to illustrate gaze paths rather than a single fixation point. The availability of prolonged gaze information allowed participants to make references to multiple locations (e.g., contrasting two different elements) and the continuous stream of gaze information made it a more salient representation compared to the zoom focus. However, many participants expressed feeling overwhelmed by the excess amount of visual information and they found it distracted from the task when working in real time.

Task Interdependence and Design

In the collaborative puzzle task the shared gaze allowed the confederate and the participant to use deictic references to quickly coordinate (figure 6 excerpt). The task was highly interdependent requiring that the pair's actions be tightly coupled which prompted an equal 


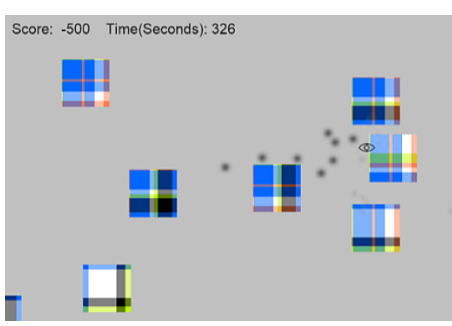

\section{Figure 6: Puzzle task and} gaze trail

Speaker 1: "it has to have the white and the green line" Speaker 2: "oh this one" Speaker 1: "okay and then maybe that one"

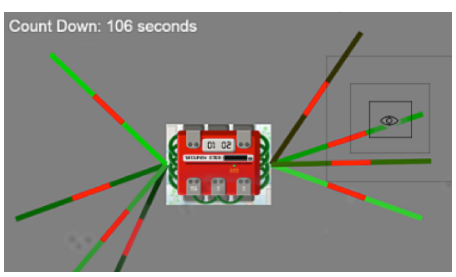

Figure 7: Bomb diffusion task and zoom focus

Director: "this one I'm looking at"

Partner: "lighter green"

Director: "yea" distribution of sending and receiving gaze information to complete the task. The consistent use of the gaze cursor as a referential pointer foregrounded the cursor and the highlighting features did not disrupt performance. In contrast, in the bomb diffusion task, the shared gaze was primarily used uni-directionally by the director, to narrow the field of possible options and make simpler comparisons. The participant would attend to the gaze signal and clarify with a brief description before acting (figure 7 excerpt).

\section{Design Implications}

Results from our interviews suggest that participants felt that highlighting gaze information in real time was disruptive. However, behavior during the task indicates that shared gaze information facilitated coordination. We designed the features of our collaborative tasks such as complex and dynamic visual spaces and highly interdependent tasks structures - to accentuate the potential benefits of shared gaze, and we see that they interact to a degree. Therefore, future work should consider task structure and design when integrating gaze information in collaborative work.

Future designs should consider less disruptive gaze representations such as partially available gaze information. For example, automatic detection of deictic references could be used to inform when to display gaze information. Additionally, while introducing time sensitivity into the task motivates efficient coordination it can also discourage engagement with unfamiliar features. Gaze awareness is still a very novel feature and not initially intuitive. Therefore, training may help improve perceptions of shared gaze and increase its effectiveness in remote collaborative work.

\section{Acknowledgements}

We thank our participants and acknowledge the support of NSF grant \#0953943 and NSF GRFP\# DGE-1324585.

\section{References}

1. Susan E. Brennan, Xin Chen, Christopher A. Dickinson, Mark B. Neider, and Gregory J. Zelinsky. 2008. Coordinating cognition: The costs and benefits of shared gaze during collaborative search. Cognition 106, 3: 1465-1477.

2. Darren Gergle, Robert E. Kraut, and Susan R. Fussell. 2006. The impact of delayed visual feedback on collaborative performance. Proceedings of the SIGCHI conference on Human Factors in computing systems, ACM, 1303-1312.

3. Bertrand Schneider and Roy Pea. 2013. Real-time mutual gaze perception enhances collaborative learning and collaboration quality. International Journal of Computer-Supported Collaborative Learning 8, 4: 375-397.

4. Kshitij Sharma, Patrick Jermann, and Pierre Dillenbourg. 2015. Displaying Teacher" s Gaze in a MOOC: Effects on Students" Video Navigation Patterns. Proceedings of the 10th European Conference on Technology Enhanced Learning.

5. Srinivas Sridharan, Reynold Bailey, Ann McNamara, and Cindy Grimm. 2012. Subtle gaze manipulation for improved mammography training. Proceedings of the Symposium on Eye Tracking Research and Applications, ACM, 75-82.

6. Randy Stein and Susan E. Brennan. 2004. Another person's eye gaze as a cue in solving programming problems. Proceedings of the 6th international conference on Multimodal interfaces, ACM, 9-15. 